\title{
Cell cycle-related kinase in carcinogenesis (Review)
}

\author{
YE TIAN, HAN WAN and GUANG TAN
}

Department of General Surgery, First Affiliated Hospital, Dalian Medical University, Dalian, Liaoning 116011, P.R. China

Received July 5, 2012; Accepted July 24, 2012

DOI: $10.3892 / \mathrm{ol} .2012 .828$

\begin{abstract}
Cell cycle-related kinase (CCRK) is a novel protein kinase homologous to both cyclin-dependent kinase 7 (Cdk7) and Cak1p groups of CDK-activating kinase (CAK). CCRK activates $\mathrm{Cdk} 2$, which controls the cell-cycle progression by phosphorylating a threonine residue conserved in $\mathrm{Cdk} 2$. Previous studies have indicated that the CCRK protein levels were elevated by more than 1.5 -fold in tumor tissue, and that the overexpression of CCRK is associated with poor prognosis of the patients. Moreover, recent studies have shown that CCRK is involved in the Wnt signaling pathway associated with the genesis and evolution of cancer. This review aims to systematically present the information currently available on CCRK obtained from in vitro and in vivo studies and highlight its significance to tumorigenesis.
\end{abstract}

\section{Contents}

1. Introduction

2. CCRK: A novel CAK in mammalian cells

3. The role of CCRK as CAK in carcinogenesis

4. The role of CCRK as a novel oncoprotein in carcinogenesis

5. CCRK as a promising target for cancer therapy

6. Conclusion

\section{Introduction}

Dysregulation of the cell cycle components may lead to tumor formation. The formation of tumors occurs when genes such as CDK, CDK-activating kinase (CAK), RB and p53 mutate, causing the cell to multiply uncontrollably (1-3). Regulation of the cell cycle progression appears to be achieved principally by cyclins and CDK activity at the G1/S and G2/M phase transi-

Correspondence to: Dr Guang Tan, Department of General Surgery, First Affiliated Hospital, Dalian Medical University, 222 Zhong Shan Lu, Dalian, Liaoning 116011, P.R. China

E-mail: lityetian@yahoo.com.cn

Key words: cyclin-dependent kinase-activating kinase, cyclin-dependent kinase 2, Wnt/ $\beta$-catenin, cyclins, male germ cellassociated kinase tions (4). The mid-G1 phase is characterized by the interaction between cyclin D1 and cdk4/6 (5). Another important complex at the G1/S boundary is that of Cdk2 and cyclin E (cyc E) (6). This complex hyperphosphorylates the retinoblastoma protein (pRb) and its family members (7). For total activation, a CDK must be binding to the subunits of cyclin, and then phosphorylated by a CAK within the activation segment or T-loop (8). Cells lacking CAKs are incapable of growth, whereas cells with a reduced level of CAKs exhibited retarded growth compared to control cells $(9,10)$. Additionally, a specific inhibition of CAKs was found to promote tumor cell death $(11,12)$.

Cell proliferation is achieved through the transition of cells from $\mathrm{G} 0 / \mathrm{G} 1$ arrest into the active cell cycle. Although progression through the cell cycle is controlled by the regulatory proteins mentioned above, overall proliferation is modulated by signaling pathways. The growth signal transduction is disrupted in almost all tumor types, as in the case of the Wnt pathway, which regulates the cell cycle mechanism to control cell proliferation and tumorigenesis $(13,14)$. Aberrant activation of the Wnt pathway occurs in several human malignancies such as hepatocellular carcinoma (HCC), gastrointestinal cancer and breast cancer $(15,16)$. Recent studies have shown that the cell cycle-related kinase (CCRK) exhibits strong oncogenic properties in HCC by activating Wnt/ $\beta$-catenin signaling and in turn upregulating the expression of $\beta$-catenin downstream targets (17).

CCRK, also known as CDK20 or p42, first reported in HeLa cells by Kaldis and Solomon in 2000 (18), is a newly identified $42 \mathrm{kDa}$ protein containing all 11 conserved subdomains characteristic of serine/threonine protein kinase (19). CCRK has sequence homology to both Cak1p and CDK7 groups of CAKs (20). Currently, CCRK is known for its role in CAK function, independently of Cdk7 complexes. CCRK is capable of phosphorylating CDK2 on Thr-160 in vitro, an intrinsic kinase activity of CDK2 that is not required for this phosphorylation $(8,11,20-22)$. Recent studies have indicated that CCRK is widely expressed in cell lines originating from a variety of tumor tissues, and CCRK suppression would cause cell cycle arrest at the G0-G1 phase by decreasing the phosphorylation of Cdk2/ Rb $(11,17,21,23)$. In addition, CCRK is overexpressed in a number of human malignancies. Mouse models of CCRK functions have provided data suggesting that the overexpression of CCRK promotes tumorigenicity $(17,23)$. Moreover, CCRK knockdown decreased the expression of cyclins and $\beta$-catenin in various types of human cancer. Therefore, CCRK phosphorylates Cdk2 and interacts with other proteins to act as an oncoprotein $(17,23)$. CCRK is 
involved in a wide array of cell signaling pathways associated with cell proliferation, which is closely linked to the genesis and evolution of cancer (17). These findings suggest that CCRK is a promising target of cancer therapy as a novel CAK or oncoprotein. In this review, we focused on the compilation of information available thus far regarding the significance of CCRK in carcinogenesis for future investigations.

\section{CCRK: A novel CAK in mammalian cells}

Cdk activation requires CAK phosphorylation of the cylin-Cdk complex on the threonine residue in the activation segment. Several lines of evidence support the role of CAK as an important cell cycle regulator, becoming a target for the design of inhibitors to regulate the cell cycle, especially in diseases such as cancer (24-27). In addition to activating Cdks, CAK regulates transcription (28). CAK associated with TFIIH phosphorylates proteins involved in transcription, including RNA polymerase II $(29,30)$. Moreover, CAK plays a role in DNA damage response. The activity of CAK associated with TFIIH decreases when DNA is damaged by UV irradiation (29). Inhibition of CAK prevents cell cycle progression, a mechanism ensuring the fidelity of chromosome transmission. The Cdk7 complex is the first and best-characterised CAK that is activated throughout the cell cycle (31). The Cdks1, 2, 4, 5 and 6 alone are inactive and require both association with a cyclin and phosphorylation on a conserved threonine residue by Cdk7 complex (32-36). However, the existence of an additional CAK, other than CDK7, has also been suggested. Kaldis and Solomon (18) biochemically enriched a 'small CAK' activity in cervical carcinoma HeLa cells. This small CAK activity is distinct in size from CDK7 activity, the former peaks at $42 \mathrm{kDa}$ whereas the latter peaks at $140 \mathrm{kDa}$ (18). Transforming growth factor (TGF) treatment in osteosarcoma U2OS cells resulted in low CDK2 Thr-160 phosphorylation without affecting CDK7 activity (37). Moreover, in D. melanogaster, inactivation of the CDK7 homolog with null and temperature-sensitive mutations showed that $\mathrm{CDK} 7$ is required for the activation of CDC2-cyclin A and CDC2-cyclin B complexes, but not for CDK2-cyc E complexes (12).

CCRK, the 'small CAK' delineated by Kaldis and Solomon (18), is a novel CAK based on: i) limited homology to Cdk7 and related kinases, with CCRK being a 346-amino acid protein with a molecular weight of $42 \mathrm{kDa}$ that shares 43\% sequence identity with CDK7. The ATP analog FSBA did not inhibit CCRK activity whereas it completely inactivated Cdk7 (19,20); ii) a modest level of CAK activity in vitro albeit weaker than CDK7 $(20,38)$. CCRK has a substrate specificity that is different from CDK7, with CCRK favoring CDK2 and MAK-related kinase/intestinal cell kinase (MRK/ICK) as the substrate $(20,29,38-40)$; iii) a cell proliferation defect, apparently accompanied by the decreasing phosphorylation of Cdk2 Thr-160, upon RNAi-mediated knockdown in human cells $(17,21,23)$. A study by Wohlbold et al (19) demonstrated that although CCRK supports colorectal carcinoma HCT116 cell or osteosarcoma U2OS cell proliferation, it does not possess CAK activity against Cdk2. Moreover, those authors found that CAK activity observed in CCRK immunoprecipitates was due to associated Cdk7 protein. The conflicting observation may be attributed to the lack of complete understanding of CCRK functions and difference in the cell lines. CCRK is known to function in both a CAK and non-CAK manner in different cell lines $(11,17,22,38)$. Thus, we provide the first evidence, to the best of our knowledge, that CCRK is a novel CAK in mammalian cells.

The main subcellular localisation of CCRK is nuclear and perinuclear regions, with a relatively low expression in the cytoplasm (23). In human tissues, CCRK is expressed predominantly in the brain and kidney, and to lesser extent in the liver, heart and placenta (20). The cardiac CCRK isoform is detectable only in heart, liver and kidney (22). In their study, Qiu et al (22) indicated that the cardiac CCRK expressed in the heart has been shown to promote cell proliferation and reduce apoptosis induced by chelerythrine. CCRK is also widely expressed in various cancer cell lines such as glioblastoma (U87, U118, U138, U373 and SW1088), cervical adenocarcinoma (HeLa), colorectal carcinoma (HCT116), osteogenic sarcoma (U2OS), breast adenocarcinoma (MCF-7), ovarian carcinoma (UACC-1598, UACC-326, OVCAR-3, HO-8910 and TOV-21G), lung fibroblast (WI-38), myoblast $(\mathrm{C} 2 \mathrm{C} 12)$ and lymphocyte (GM08336) $(20,23)$. Notably, the disruption of CCRK homeostasis is closely linked to the formation of various types of human cancer (Table I). A high expression level of CCRK is a strong and an independent predictor of short overall survival in ovarian carcinoma and HCC $(17,23)$. Thus, CCRK expression appears to have the potential to predict ovarian carcinoma and HCC patient clinical outcome (17). These data suggest that CCRK is essential for the proliferation of several human malignancies. Thus, the functions of CCRK in both a CAK and an oncoprotein manner were delineated.

\section{The role of CCRK as CAK in carcinogenesis}

Liu et al (20) were the first to show that CCRK has CAK activity that regulates cell growth. In cervical adenocarcinoma HeLa cells, the RNAi-mediated ablation of CCRK was able to inhibit cell proliferation, cause cell cycle G1 phase arrest, decrease pCdk 2 levels and inhibit Cdk 2 kinase activity (20). Similar results were observed in human glioblastoma (21), in which suppression of CCRK by small interfering RNA (siRNA) inhibited glioblastoma cell growth, induced cell cycle G1 arrest and decreased the pCdk2 level, whereas the overexpression of CCRK was able to confer tumorigenicity to a non-tumorigenic glioblastoma cell line, indicating that CCRK expression levels potentially correlate with the tumorigenicity of glioblastoma cells. In another study, An et al (11) investigated the function of CCRK in colorectal cancer carcinogenesis. Those authors showed that CCRK knockdown by siCCRK or shCCRK significantly reduces cell growth and causes cell cycle arrest at the G0-G1 phase in both LoVo and DLD1 human colorectal cancer cell lines. In addition, cyc E-Cdk2 complexes, the important complex at the G1/S boundary, reduced phosphorylation when CCRK was knocked down. Moreover, siCCRK transfection markedly reduced $\mathrm{pRb}$ phosphorylation. The $\mathrm{pRb}$ is a tumor suppressor protein that is dysfunctional in several major cancers (41). Besides, it is worth noting that knockdown of CCRK induced cell cycle arrest at the G2-M phase in the LoVo cells. 
Table I. Overexpression of CCRK in the human tumors investigated.

No.

\begin{tabular}{lccc}
$\begin{array}{l}\text { Tumor } \\
\text { type }\end{array}$ & $\begin{array}{c}\text { No. } \\
\text { investigated }\end{array}$ & $\begin{array}{c}\text { with elevated } \\
\text { CCRK }\end{array}$ & Refs. \\
\hline Hepatocellular carcinoma & 52 & 45 & $(17)$ \\
Ovarian carcinoma & 122 & 65 & $(23)$ \\
Colorectal cancer & 10 & 7 & $(11)$ \\
Glioblastoma & 19 & 14 & $(21)$
\end{tabular}

CCRK, cell cycle-related kinase.

The evidence mentioned above suggests that although the overall picture of CCRK regulation remains incomplete, various aspects can be merged to provide a perspective of the role of this kinase cell cycle disruption. In its role as a catalyst for Cdk2 activity, CCRK provokes tumor-associated cell cycle defects to induce unscheduled proliferation. Excess CCRK phosphorylates the Cdk2 on Thr-160, subsequently promoting the transition from the $\mathrm{G} 1$ to $\mathrm{S}$ phase through the phosphorylation of key target proteins, including the $\mathrm{pRb}$ as well as the Rb family members p130 and p107. The hyperphosphorylated $\mathrm{RB}$ releases transcription factors such as E2F that are complexed with pRb (42). In turn, E2F binds to and activates the promoters of genes important in DNA synthesis (43). Additionally, CCRK only rarely phosphorylates CDK2. Phosphorylation by CCRK is stimulated by the association of CDK2 with its relevant cyclin, cyc E. After cyc $\mathrm{E}$ binding, the activation segment moves towards the C-terminal lobe exposing Thr160 for CCRK phosphorylation (43-45). In colorectal cancer cell lines, CCRK knockdown decreases the phosphorylation of $\mathrm{Cdk} 2 / \mathrm{Rb}$ and reduces the expression of cyc E, suggesting that CCRK interacts with cyc E to upregulate the expression of the latter, enabling CCRK to phosphorylate CDK2 (11) (Fig. 1).

A similar regulatory mechanism has been observed whereby CCRK activates human MAK (male germ cellassociated kinase) and MRK/ICK by phosphorylation of the essential Thr-157 in the T-loop $(38,46)$. Both MAK and MRK reportedly phosphorylate Scythe, an antiapoptotic protein required for apoptosis and proliferation during mammalian development, in vitro (47-49). Recent studies have shown that MRK/MAK are new downstream targets of protein phosphatase 5 (PP5), which plays a crucial role in biological functions and controls almost every cell process, including gene transcription and translation, cell-cycle progression, DNA damage response and proliferation. Inhibition of PP5 expression leads to a marked antiproliferative effect by activation of the p53-dependent G1 checkpoint $(50,51)$. These findings suggest that MRK and/or MAK play a key role in cell-cycle checkpoint control and apoptosis in response to stress conditions, such as DNA damage. Moreover, the phenotypic effects of silencing CCRK in malignant cells may channel through MRK and/or MAK (52). Detailed investigations are essential to confirm the existence of the relationship between CCRK and MRK/MAK.

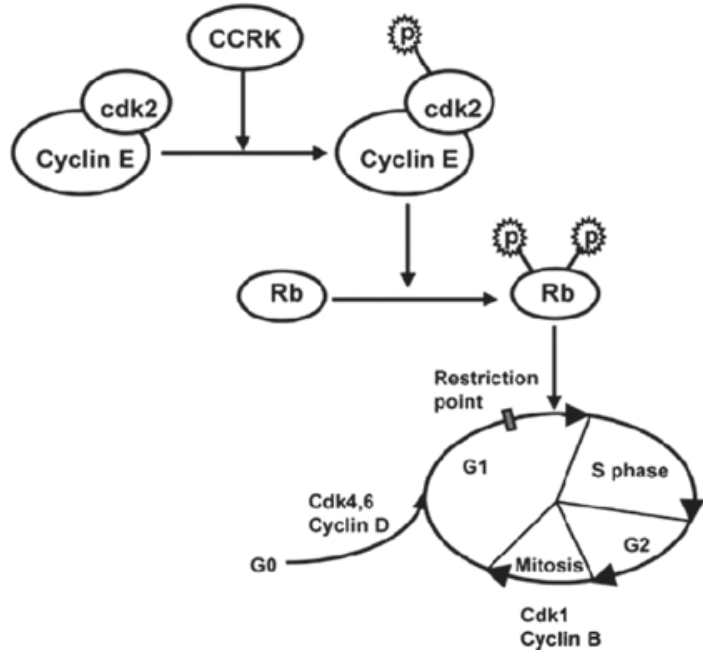

Figure 1. Cell cycle-related kinase (CCRK) serves as a novel oncogenic CDK-activating kinase (CAK) in cell cycle control and carcinogenesis.

\section{Role of CCRK as a novel oncoprotein in carcinogenesis}

In addition to phosphorylating and activating CDK2, CCRK possesses an alternative mechanism for CCRK-induced cell cycle progression and cell proliferation. Previous studies have shown that CCRK is involved in a wide array of cell signaling pathways such as the Wnt and androgen receptor (AR) signaling pathways. The Wnt signaling pathway is a network of proteins best known for its role in embryogenesis and cancer $(13,14)$. Aberrant activation of the Wnt signaling pathway occurs in several human cancers such as HCC, gastrointestinal cancer and breast cancer $(15,16)$. Advances in molecular research have demonstrated that AR signaling is involved in key cell processes such as proliferation and apoptosis. Accumulating evidence has shown that AR is a master regulator in the G1/S phase progression in cancer cells and the crosstalk between this ligand-activated transcription factor and cell cycle pathways $(53,54)$.

It was reported that CCRK links the AR and Wnt/ $\beta$-catenin/TCF cascades and provides a mechanistic basis for the aberrant $\beta$-catenin activation in human HCCs (17). $\beta$-catenin is a subunit of the cadherin protein complex and has been suggested as the most important integral component in the Wnt signaling pathway $(55,56)$. Upon activation, $\beta$-catenin is able to escape from the destruction complex comprising APC, Axin and glycogen synthase kinase-3 $\beta$ (GSK-3 $\beta$ ), which phosphorylates $\beta$-catenin and targets it for degradation via the ubiquitin-mediated proteasomal pathway $(57,58)$. The excess cytoplasmic $\beta$-catenin is subsequently translocated into the nucleus to perform a variety of functions such as cell proliferation, the first step in cancer (59-61). It can act in conjunction with TCF and LEF to regulate the expression of numerous key target genes such as epidermal growth factor receptor (EGFR) (62). In their study, Feng et al (17) showed that CCRK expression in immortal liver cells induced focus formation, anchorage-independent growth, and tumor formation in immunodeficient mice, whereas CCRK knockdown in HCC cells reduced G1/S phase progression, focus formation, and tumorigenicity, demonstrating the strong oncogenic capacity of CCRK in HCC. Moreover, $\beta$-catenin depletion 


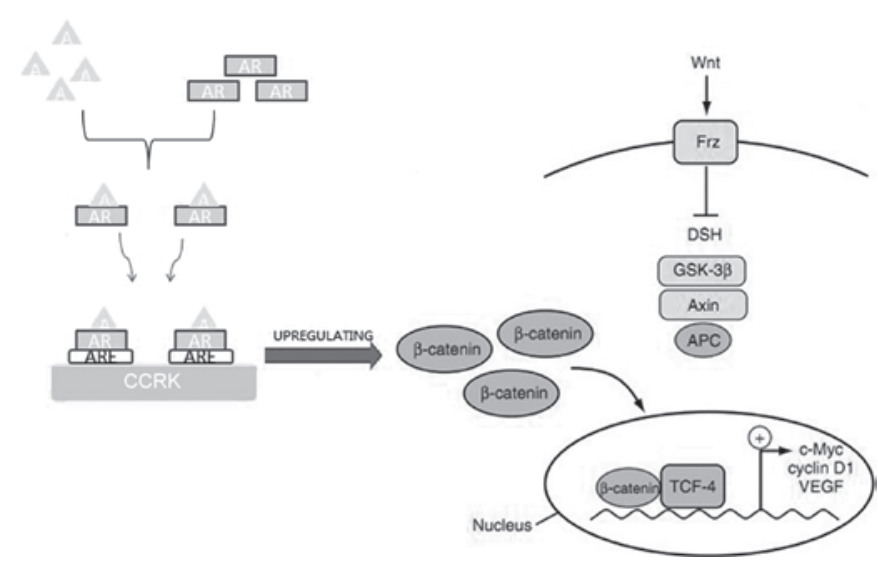

Figure 2. In the presence of cell cycle-related kinase (CCRK) overexpression, $\beta$-catenin is stabilized, and excess cytoplasmic $\beta$-catenin is translocated to the nucleus, where it interacts with the TCF-4 transcription factor to regulate the expression of a number of key target genes.

reverses the CCRK-induced tumorigenicity in nude mice, indicating that active $\beta$-catenin signaling is a major mediator of CCRK-induced tumorigenicity. These data are in agreement with the fact that AR silencing reduced the expression of active $\beta$-catenin and $\beta$-catenin target genes, which could be rescued by ectopic CCRK expression. Furthermore, the CCRK expression correlates with AR and active $\beta$-catenin levels in primary HCC specimens. Therefore, a vicious cycle exists: AR induces CCRK expression to stimulate $\beta$-catenin activity, while $\beta$-catenin, acting downstream of CCRK, induces AR expression and activity. CCRK may therefore be a positive component in the Wnt signaling pathway, present in the cell to aberrantly activate $\beta$-catenin, and to provoke tumor-associated cell cycle defects to induce unscheduled proliferation (Fig. 2).

The mechanism underlying $\beta$-catenin activation by CCRK remains unclear. CCRK is potentially able to dysregulate $\beta$-catenin activation. Firstly, CCRK may phosphorylate GSK3 $\beta$ at Thr390. Recent studies have suggested that the C-terminal of GSK3ß, specifically phosphorylation at Thr390, is important for $\beta$-catenin activation (63). Secondly, CCRK may directly interact with the N-terminal domain of $\beta$-catenin, allowing $\beta$-catenin to escape from its negative control. Thirdly, CCRK may functionally interact with TCF, leading to the transcription of target genes through the interaction of $\beta$-catenin with TCF (64). However, further investigation is needed to validate these hypotheses. Since AR and Wnt signaling pathways are important in various types of human cancer and CCRK overexpression was detected in a number of cancer cells, the disruption of the AR-CCRK- $\beta$-catenin-positive regulatory circuit is highly relevant to cancer formation in $\mathrm{HCC}$, and potentially to other male-predominant cancers.

Besides modulating the signaling pathway involved in cell cycle control and cell proliferation, CCRK has also been shown to regulate cyclin D1 expression. Wu et al (23) observed that CCRK overexpression in TOV-21G (ovarian carcinoma) cells induced an upregulated expression of cyclin D1, but not in Cdk2, pCdk2, Cdk4 and Cdk6 molecules, the results suggesting that the mechanism by which CCRK regulates cell cycle progression may be cell-type specific. In both the HCC and ovarian carcinoma cell lines, CCRK overexpression caused the upregulated expression of cyclin D1 $(17,23)$.
Consistently, RNA interference and transfection studies have shown that silencing of CCRK reduced the cyclin D1 expression both in mRNA and in protein levels, and inhibited HCC and ovarian carcinoma cell proliferation both in vitro and in vivo. In addition, a significant correlation between the expression of CCRK and cyclin D1 in ovarian carcinomas was observed. Studies have also demonstrated that knockdown of $\beta$-catenin abrogated the CCRK-induced cyclin D1 expression and G1/S phase transition of liver cells. The overexpression of cyclin D1 was found to often associate closely with incessant tumor growth, progression and/or poor patient outcome. Thus, abnormally high levels of cyclin D1 are involved in a number of GI and non-GI malignancies, including those originating from the oral cavity, esophagus, breast and bladder (65-67). Therefore, investigation into the molecular mechanisms and signal transduction pathways between CCRK and cyclin D1 in various types of cancer is crucial.

\section{CCRK as a promising target for cancer therapy}

CCRK was overexpressed in various types of cancer (Table I). Increasing levels of CCRK were correlated with poor survival in patients with HCC (17). Furthermore, a high expression of CCRK was associated with poor prognosis in ovarian carcinoma (23). Increased CCRK expression affects brain tumorigenesis by upregulating CDK2 activity (21). The suppression of CCRK by siCCRK was found to induce G1 phase cell cycle arrest and reduced cell growth of colorectal carcinoma cells (11). Consistently, stable clones of colorectal carcinoma cells expressing shCCRK exhibited decreased cell proliferation rates (11). CCRK is also involved in the human papillomavirus association with cervical cancer (20). Moreover, CCRK is able to regulate AR and $\beta$-catenin activity, which are closely associated with various types of cancer $(14,17)$. As disruption of the cell cycle control is a hallmark of all malignant cells, CCRK is potentially an important cell cycle regulator during carcinogenesis. The association between CCRK and CDK2 is well characterised at the structural and biological levels. Several lines of evidence strongly suggest that CCRK positively regulates cell cycle progression and tumor growth, indicating that CCRK may serve as a novel prognostic marker and may be a promising candidate as a molecular target for cancer therapy for some types of cancer. The use of molecule inhibitors to block the action of CCRK has not been previously reported.

\section{Conclusion}

Previous data have shown that CCRK is a novel CAK and serves as an important regulator in tumorigenicity. CCRK is functionally connected to a broad range of cell signaling pathways with important functions in cell cycle progression, cell proliferation and malignant transformation. The involvement of CCRK in tumorigenesis is extensive. Numerous activities of CCRK affect tumor growth, both in a CAK and an oncoprotein manner.

Currently, the best understood function of CCRK is its role in phosphorylating cdk2 on Thr-160. However, several questions concerning the function of CCRK in this pathway remain. It is possible that siCCRK-reduced CDK2 phosphorylation is a secondary effect of the growth inhibition induced 
by CCRK knockdown. In addition, CCRK may bind and/or phosphorylate CDK7 (or other CDKs), which in turn bind to CDK2 and regulate its activity (21). However, more studies such as in vitro kinase assays are needed to confirm whether CCRK interacts directly with CDK2. In addition, previous studies suggest that the T-loop phosphorylation of Cdk2 is completely dispensable (68). Members of the Ringo/speedy family proteins have now been shown to associate with Cdk2 and promote its activity against some substrates where T-loop phosphorylation has no effect on the Cdk2 activity (69). Future studies on the structure of Ringo/Cdk 2 complexes and more thorough genetic and biochemical studies on $\mathrm{Cdk} 2$ and CCRK are required to elucidate these issues. It is also worth investigating the relationship between CCRK and MRK/ MAK, the findings of which may reveal novel mechanisms for cell cycle regulation.

CCRK is also capable of interacting with $\beta$-catenin as a positive component in the Wnt signaling pathway. Future studies are needed to elucidate the precise mechanism of action of CCRK in regulating $\beta$-catenin. Disruption of the AR-CCRK- $\beta$-catenin-positive regulatory circuit plays a critical role in hepatocarcinogenesis, and may be highly relevant to the design of therapeutic interventions in some types of cancer (17). Furthermore, the upregulated expression of CCRK in ovarian carcinoma and HCC suggests a potentially important role of CCRK in the control of cell proliferation via the regulation of cyclin D1 expression $(17,23)$.

The ultimate aim of these studies is to translate them into clinical applications. If its role as an important regulator in tumorigenicity is confirmed, CCRK may serve as a novel prognostic cancer marker and a new target of cancer therapeutics.

\section{References}

1. Green DR and Evan GI: A matter of life and death. Cancer Cell 1: 19-30, 2002.

2. Zwang Y, Oren M and Yarden Y: Consistency test of the cell cycle: roles for $\mathrm{p} 53$ and EGR1. Cancer Res 72: 1051-1054, 2012.

3. Cicenas J and Valius M: The CDK inhibitors in cancer research and therapy. J Cancer Res Clin Oncol 137: 1409-1418, 2011.

4. Pines J: Four-dimensional control of the cell cycle. Nat Cell Biol 1: E73-E79, 1999.

5. Ortega S, Malumbres M and Barbacid M: Cyclin D-dependent kinases, INK4 inhibitors and cancer. Biochim Biophys Acta 1602: 73-87, 2002.

6. Mitra K, Wunder C, Roysam B, Lin G and Lippincott-Schwartz J: A hyperfused mitochondrial state achieved at G1-S regulates cyclin E buildup and entry into S phase. Proc Natl Acad Sci USA 106: 11960-11965, 2009.

7. Cobrinik D: Pocket proteins and cell cycle control. Oncogene 24: 2796-2809, 2005.

8. Lolli G and Johnson LN: CAK-cyclin-dependent activating kinase: a key kinase in cell cycle control and a target for drugs? Cell Cycle 4: 572-577, 2005.

9. Sutton A and Freiman R: The Cak1p protein kinase is required at G1/S and G2/M in the budding yeast cell cycle. Genetics 147: 57-71, 1997.

10. Wallenfang MR and Seydoux G: cdk-7 is required for mRNA transcription and cell cycle progression in Caenorhabditis elegans embryos. Proc Natl Acad Sci USA 99: 5527-5532, 2002.

11. An X, Ng SS, Xie D, et al: Functional characterisation of cell cycle-related kinase (CCRK) in colorectal cancer carcinogenesis. Eur J Cancer 46: 1752-1761, 2010.

12. Larochelle S, Pandur J, Fisher RP, Salz HK and Suter B: Cdk7 is essential for mitosis and for in vivo Cdk-activating kinase activity. Genes Dev 12: 370-381, 1998.

13. Clevers H: Wnt/beta-catenin signaling in development and disease. Cell 127: 469-480, 2006.
14. MacDonald BT, Tamai $\mathrm{K}$ and He X: Wnt/beta-catenin signaling: components, mechanisms, and diseases. Dev Cell 17: 9-26, 2009.

15. Yang W, Yan HX, Chen L, et al: Wnt/beta-catenin signaling contributes to activation of normal and tumorigenic liver progenitor cells. Cancer Res 68: 4287-4295, 2008.

16. Ying Y and Tao Q: Epigenetic disruption of the WNT/betacatenin signaling pathway in human cancers. Epigenetics 4: 307-312, 2009.

17. Feng H, Cheng AS, Tsang DP, et al: Cell cycle-related kinase is a direct androgen receptor-regulated gene that drives betacatenin/T cell factor-dependent hepatocarcinogenesis. J Clin Invest 121: 3159-3175, 2011

18. Kaldis P and Solomon MJ: Analysis of CAK activities from human cells. Eur J Biochem 267: 4213-4221, 2000.

19. Wohlbold L, Larochelle S, Liao JC, Livshits G, Singer J, Shokat KM and Fisher RP: The cyclin-dependent kinase (CDK) family member PNQALRE/CCRK supports cell proliferation but has no intrinsic CDK-activating kinase $(\mathrm{CAK})$ activity. Cell Cycle 5: 546-554, 2006.

20. Liu Y, Wu C and Galaktionov K: p42, a novel cyclin-dependent kinase-activating kinase in mammalian cells. J Biol Chem 279: 4507-4514, 2004.

21. Ng SS, Cheung YT, An XM, et al: Cell cycle-related kinase: a novel candidate oncogene in human glioblastoma. J Natl Cancer Inst 99: 936-948, 2007.

22. Qiu H, Dai H, Jain K, Shah R, Hong C, Pain J, Tian B, Vatner DE, Vatner SF and Depre C: Characterization of a novel cardiac isoform of the cell cycle-related kinase that is regulated during heart failure. J Biol Chem 283: 22157-22165, 2008.

23. Wu GQ, Xie D, Yang GF, Liao YJ, Mai SJ, Deng HX, Sze J, Guan XY, Zeng YX, Lin MC and Kung HF: Cell cycle-related kinase supports ovarian carcinoma cell proliferation via regulation of cyclin D1 and is a predictor of outcome in patients with ovarian carcinoma. Int J Cancer 125: 2631-2642, 2009.

24. Yang Y, Hu Y, Gu HY, Lu N, Liu W, Qi Q, Zhao L, Wang XT, You QD and Guo QL: Oroxylin A induces G2/M phase cell-cycle arrest via inhibiting Cdk7-mediated expression of Cdc2/p34 in human gastric carcinoma BGC-823 cells. J Pharm Pharmacol 60: 1459-1463, 2008

25. Yu J, Guo QL, You QD, Zhao L, Gu HY, Yang Y, Zhang HW, Tan $\mathrm{Z}$ and Wang X: Gambogic acid-induced G2/M phase cellcycle arrest via disturbing CDK7-mediated phosphorylation of $\mathrm{CDC} 2 / \mathrm{p} 34$ in human gastric carcinoma BGC-823 cells. Carcinogenesis 28: 632-638, 2007.

26. Krystof V and Uldrijan S: Cyclin-dependent kinase inhibitors as anticancer drugs. Curr Drug Targets 11: 291-302, 2010.

27. Węsierska-Gądek $J$ and Maurer M: Promotion of apoptosis in cancer cells by selective purine-derived pharmacological CDK inhibitors: one outcome, many mechanisms. Curr Pharm Des 17: 256-271, 2011.

28. Egly JM and Coin F: A history of TFIIH: two decades of molecular biology on a pivotal transcription/repair factor. DNA Repair (Amst) 10: 714-721, 2011.

29. Patel SA and Simon MC: Functional analysis of the Cdk7.cyclin H.Mat complex in mouse embryonic stem cells and embryos. J Biol Chem 285: 15587-15598, 2010.

30. Glover-Cutter K, Larochelle S, Erickson B, Zhang C, Shokat K, Fisher RP and Bentley DL: TFIIH-associated Cdk7 kinase functions in phosphorylation of C-terminal domain Ser7 residues, promoter-proximal pausing, and termination by RNA polymerase II. Mol Cell Biol 29: 5455-5464, 2009.

31. Fisher RP: Secrets of a double agent: CDK7 in cell-cycle control and transcription. J Cell Sci 118: 5171-5180, 2005.

32. Larochelle S, Merrick KA, Terret ME, Wohlbold L, Barboza NM, Zhang C, Shokat KM, Jallepalli PV and Fisher RP: Requirements for Cdk7 in the assembly of Cdk1/cyclin B and activation of Cdk2 revealed by chemical genetics in human cells. Mol Cell 25: 839-850, 2007.

33. Pillai P, Desai S, Patel R, Sajan M, Farese R, Ostrov D and Acevedo-Duncan M: A novel PKC-iota inhibitor abrogates cell proliferation and induces apoptosis in neuroblastoma. Int J Biochem Cell Biol 43: 784-794, 2011.

34. Bica L, Meyerowitz J,Parker SJ, Caragounis A, Du T,Paterson BM, Barnham KJ, Crouch PJ, White AR and Donnelly PS: Cell cycle arrest in cultured neuroblastoma cells exposed to a bis(thiosemicarbazonato) metal complex. Biometals 24: 117-133, 2011.

35. Fisher RP and Morgan DO: A novel cyclin associates with MO15/ CDK7 to form the CDK-activating kinase. Cell 78: 713-724, 1994

36. Lolli G and Johnson LN: Recognition of Cdk2 by Cdk7. Proteins 67: 1048-1059, 2007. 
37. Nagahara H, Ezhevsky SA, Vocero-Akbani AM, Kaldis P, Solomon MJ and Dowdy SF: Transforming growth factor beta targeted inactivation of cyclin E:cyclin-dependent kinase 2 (Cdk2) complexes by inhibition of Cdk2 activating kinase activity. Proc Natl Acad Sci USA 96: 14961-14966, 1999.

38. Fu Z, Larson KA, Chitta RK, et al: Identification of yin-yang regulators and a phosphorylation consensus for male germ cell-associated kinase (MAK)-related kinase. Mol Cell Biol 26 : 8639-8654, 2006.

39. Marshall RM and Grana X: Mechanisms controlling CDK9 activity. Front Biosci 11: 2598-2613, 2006.

40. Yang WH, Heaton JH, Brevig H, Mukherjee S, Iñiguez-Lluhí JA and Hammer GD: SUMOylation inhibits SF-1 activity by reducing CDK7-mediated serine 203 phosphorylation. Mol Cell Biol 29: 613-625, 2009

41. Sachdeva UM and O'Brien JM: Understanding pRb: toward the necessary development of targeted treatments for retinoblastoma. J Clin Invest 122: 425-434, 2012.

42. Kolupaeva V and Basilico C: Overexpression of cyclin E/ CDK2 complexes overcomes FGF-induced cell cycle arrest in the presence of hypophosphorylated $\mathrm{Rb}$ proteins. Cell Cycle: Jul. 1, 2012 (E-pub ahead of print).

43. Meraldi P, Lukas J, Fry AM, Bartek J and Nigg EA: Centrosome duplication in mammalian somatic cells requires E2F and Cdk2-cyclin A. Nat Cell Biol 1: 88-93, 1999.

44. Stamatakos M, Palla V, Karaiskos I, Xiromeritis K, Alexiou I, Pateras I and Kontzoglou K: Cell cyclins: triggering elements of cancer or not? World J Surg Oncol 8: 111, 2010.

45. Weinberg RA: The retinoblastoma protein and cell cycle control. Cell 81: 323-330, 1995.

46. Xia L, Robinson D, Ma AH, Chen HC, Wu F, Qiu Y and Kung HJ: Identification of human male germ cell-associated kinase, a kinase transcriptionally activated by androgen in prostate cancer cells. J Biol Chem 277: 35422-35433, 2002.

47. Desmots F, Russell HR, Lee Y, Boyd K and McKinnon PJ: The reaper-binding protein scythe modulates apoptosis and proliferation during mammalian development. Mol Cell Biol 25 10329-10337, 2005.

48. Desmots F, Russell HR, Michel D and McKinnon PJ: Scythe regulates apoptosis-inducing factor stability during endoplasmic reticulum stress-induced apoptosis. J Biol Chem 283: 3264-3271, 2008

49. Minami R, Shimada M, Yokosawa $\mathrm{H}$ and Kawahara $\mathrm{H}$ : Scythe regulates apoptosis through modulating ubiquitinmediated proteolysis of the Xenopus elongation factor XEF1AO. Biochem J 405: 495-501, 2007.

50. Golden T, Swingle M and Honkanen RE: The role of serine/ threonine protein phosphatase type 5 (PP5) in the regulation of stress-induced signaling networks and cancer. Cancer Metastasis Rev 27: 169-178, 2008.

51. Ham BM, Jayachandran H, Yang F, et al: Novel Ser/Thr protein phosphatase 5 (PP5) regulated targets during DNA damage identified by proteomics analysis. J Proteome Res 9: 945-953, 2010.
52. Ma AH, Xia L, Desai SJ, Boucher DL, Guan Y, Shih HM, Shi XB, deVere White RW, Chen HW, Tepper CG and Kung HJ: Male germ cell-associated kinase, a male-specific kinase regulated by androgen, is a coactivator of androgen receptor in prostate cancer cells. Cancer Res 66: 8439-8447, 2006.

53. Balk SP and Knudsen KE: AR, the cell cycle, and prostate cancer. Nucl Recept Signal 6: e001, 2008.

54. Kalra M, Mayes J, Assefa S, Kaul AK and Kaul R: Role of sex steroid receptors in pathobiology of hepatocellular carcinoma. World J Gastroenterol 14: 5945-5961, 2008.

55. Amin $\mathrm{N}$ and Vincan E: The Wnt signaling pathways and cell adhesion. Front Biosci 17: 784-804, 2012.

56. Tarapore RS, Siddiqui IA and Mukhtar H: Modulation of Wnt/ beta-catenin signaling pathway by bioactive food components. Carcinogenesis 33: 483-491, 2012

57. Kimelman $\mathrm{D}$ and $\mathrm{Xu} \mathrm{W}$ : beta-catenin destruction complex: insights and questions from a structural perspective. Oncogene 25 7482-7491, 2006.

58. Roberts DM, Pronobis MI, Poulton JS, Waldmann JD, Stephenson EM, Hanna S and Peifer M: Deconstructing the $\beta$-catenin destruction complex: mechanistic roles for the tumor suppressor APC in regulating Wnt signaling. Mol Biol Cell 22: 1845-1863, 2011.

59. Kikuchi A, Yamamoto H, Sato A and Matsumoto S: New insights into the mechanism of Wnt signaling pathway activation. Int Rev Cell Mol Biol 291: 21-71, 2011.

60. Logan CY and Nusse R: The Wnt signaling pathway in development and disease. Annu Rev Cell Dev Biol 20: 781-810, 2004.

61. Whittaker S, Marais R and Zhu AX: The role of signaling pathways in the development and treatment of hepatocellular carcinoma. Oncogene 29: 4989-5005, 2010.

62. Tan X, Apte U, Micsenyi A, Kotsagrelos E, Luo JH, Ranganathan S, Monga DK, Bell A, Michalopoulos GK and Monga SP: Epidermal growth factor receptor: a novel target of the Wnt/beta-catenin pathway in liver. Gastroenterology 129: 285-302, 2005

63. Buescher JL and Phiel CJ: A noncatalytic domain of glycogen synthase kinase-3 (GSK-3) is essential for activity. J Biol Chem 285: 7957-7963, 2010.

64. Uysal-Onganer P and Kypta RM: Wnt11 in 2011 - the regulation and function of a non-canonical Wnt. Acta Physiol (Oxf) 204: 52-64, 2012.

65. Gordon V, Bhadel S, Wunderlich W, et al: CDK9 regulates AR promoter selectivity and cell growth through serine 81 phosphorylation. Mol Endocrinol 24: 2267-2280, 2010.

66. Kim JK and Diehl JA: Nuclear cyclin D1: an oncogenic driver in human cancer. J Cell Physiol 220: 292-296, 2009.

67. Musgrove EA, Caldon CE, Barraclough J, Stone A and Sutherland RL: Cyclin D as a therapeutic target in cancer. Nat Rev Cancer 11: 558-572, 2011.

68. Nebreda AR: CDK activation by non-cyclin proteins. Curr Opin Cell Biol 18: 192-198, 2006.

69. Abbas T and Dutta A: CDK2-activating kinase (CAK): more questions than answers. Cell Cycle 5: 1123-1124, 2006. 